\title{
Obesidade em paciente com prognóstico de gravidade para Covid-19
}

\author{
Obesity in patient with prognosis of gravity for Covid-19 \\ Obesidad en paciente con pronóstico de embarazo por Covid-19
}

Recebido: 05/08/2021 | Revisado: 10/08/2021 | Aceito: 12/08/2021 | Publicado: 15/08/2021

José Auricélio Bernardo Cândido

ORCID: https://orcid.org/0000-0003-3327-8861

Prefeitura Municipal de Horizonte, Brasil E-mail: jabcauricelio60@hotmail.com

Maria Rosilene Cândido Moreira

ORCID: https://orcid.org/0000-0002-9821-1935 Universidade Federal do Cariri, Brasil E-mail: rosilene.moreira@ufca.edu.br Severino Ferreira Alexandre ORCID: https://orcid.org/0000-0002-6047-289X Secretaria da Saúde do Ceará, Brasil E-mail: linoal@gmail.com

Natália Campos Parente

ORCID: https://orcid.org/0000-0002-5866-1157 Secretaria Municipal de Saúde de Crato, Brasil E-mail: natyparente@ hotmail.com

Naara Regia Pinheiro Cavalcante ORCID: https://orcid.org/0000-0003-0261-9168 Secretaria da Saúde do Ceará, Brasil E-mail: naarapinheiro@gmail.com

\section{Resumo}

A COVID-19 é uma doença viral de alta transmissibilidade que pode causar desconforto respiratório agudo e evoluir para falência de vários órgãos. Percebe-se há evidencias fisiopatológicas semelhantes entre a obesidade e a COVID. Relato de caso com análise documental por meio da revisão do prontuário, relatórios de evolução médica e de Enfermagem, exames laboratoriais e Tomografia Computadorizada, durante o período de internamento do paciente com obesidade em um Hospital Público. Neste relato de caso, apresentamos o escopo multifacetado da COVID-19, que afetou um paciente adulto jovem, apresentando obesidade grau II como única comorbidade. Os dados do caso indicam uma relação entre a comorbidade e a doença, incluindo seu prognóstico e tempo de internação. A infecção COVID-19 teve um comportamento persistente. A obesidade, como a única comorbidade detectada, pareceu contribuir para o retardo, da recuperação e da alta hospitalar. É importante relatar casos sobre o novo coronavírus para que a comunidade médica possa explorar e aprofundar as experiências em torno dessa doença. Estudos futuros devem apontar para evidências concretas sobre a relação entre obesidade e COVID-19.

Palavras-chave: COVID-19; Obesidade; Prognóstico.

\begin{abstract}
COVID-19 is a highly transmissible viral disease that can cause acute respiratory distress and progress to multiple organ failure. It is noticed that there is similar pathophysiological evidence between obesity and COVID. Case report with documental analysis through medical record review, medical and nursing evolution reports, laboratory tests and CT scan, during the hospitalization period of the patient with obesity in a Public Hospital. In this case report, we present the multifaceted scope of COVID-19, which affected a young adult patient, with grade II obesity as the only comorbidity. The case data indicate a relationship between comorbidity and the disease, including its prognosis and length of stay. The COVID-19 infection had a persistent behavior. Obesity, as the only comorbidity detected, seemed to contribute to delay, recovery and hospital discharge. It is important to report cases about the new coronavirus so that the medical community can explore and deepen the experiences surrounding this disease. Future studies should point to concrete evidence on the relationship between obesity and COVID-19.
\end{abstract}

Keywords: COVID-19; Obesity; Prognosis.

\section{Resumen}

COVID-19 es una enfermedad viral altamente transmisible que puede causar dificultad respiratoria aguda y progresar a insuficiencia multiorgánica. Se observa que existe evidencia fisiopatológica similar entre obesidad y COVID. Reporte de caso con análisis documental mediante revisión de historia clínica, informes de evolución médica y de enfermería, pruebas de laboratorio y tomografía computarizada, durante el período de internación del paciente con obesidad en un Hospital Público. En este caso clínico, presentamos el alcance polifacético del COVID-19, que afectó 
a un paciente adulto joven, con obesidad grado II como única comorbilidad. Los datos de los casos indican una relación entre la comorbilidad y la enfermedad, incluido su pronóstico y la duración de la estancia. La infección por COVID-19 tuvo un comportamiento persistente. La obesidad, como única comorbilidad detectada, parece contribuir al retraso, la recuperación y el alta hospitalaria. Es importante reportar casos sobre el nuevo coronavirus para que la comunidad médica pueda explorar y profundizar las experiencias en torno a esta enfermedad. Los estudios futuros deberían apuntar a evidencia concreta sobre la relación entre la obesidad y COVID-19.

Palabras clave: COVID-19; Obesidad; Pronóstico.

\section{Introdução}

A COVID-19 é uma doença causada pelo vírus causador Síndrome Respiratória Aguda Grave (SARS-COV-2), identificado inicialmente na cidade de Wuhan na China. O vírus possui alta transmissibilidade e podem provocar desconforto respiratório agudo e progredir para a falência de vários órgãos (McIntosh, 2020). Estima-se que a doença possa levar ao aparecimento de casos leves ou assintomáticos (80\%) e casos graves (20\%) que podem requerer atendimento hospitalar por apresentar insuficiência respiratória. Destes, $5 \%$ podem necessitar de suporte ventilatório. Sua letalidade pode variar conforme a faixa etária e as condições clínicas associadas (Brasil, 2020).

As comorbidades mais evidenciadas em relação à COVID-19 foram as doenças crônicas como hipertensão arterial, diabetes mellitus, doenças coronarianas, doença renal e pulmonar crônica, e câncer (Guan et. al., 2020). Pacientes sem comorbidades apresentaram baixa letalidade para a doença $(0,9 \%)$, enquanto que os pacientes com comorbidades tiveram taxa de letalidade mais elevadas variando entre 6,5\% e 10,5\% (Wu \& McGoogan, 2020).

A obesidade é uma comorbidade que recentemente vem sendo estudada por pesquisadores em relação à COVID-19. Percebe-se há evidencias fisiopatológicas semelhantes entre a obesidade e a COVID como a diminuição de reserva expiratória, a capacidade funcional, a complacência do sistema respiratório, a excursão diafragmática e o aumento de citocinas inflamatórias, que podem contribuir para a morbidade da doença (Dietz \& Santos-Burgoa, 2020).

A adiposidade presente em pacientes obesos proporciona uma inflamação crônica devido à resistência à insulina sistêmica que vai desencadear um grande processo inflamatório aumentando a vulnerabilidade a infecções. A grande quantidade de adipocinas inflamatórias liberadas pela concentração de gorduras no organismo vai interferir na resposta imune juntamente com as citocinas liberadas pela presença do SARS-COV-2 (Kassir, 2020). A obesidade em si pode aumentar o risco de trombose que, associado à COVID-19 pode desencadear coagulação intravascular disseminada e tromboembolismo venoso. Essas situações requerem tratamento intensivo imediato (Sattar, McInnes \& McMurray, 2020).

Diante dos processos inflamatórios, alguns marcadores sanguíneos em exames laboratoriais ficam mais evidentes como a linfocitopenia, níveis altos de neutrófilos, da Proteína C Reativa, do HDL, do dímero D, de uréia e creatinina (Zavascki \& Falci, 2020). A Tomografia Computadorizada evidencia opacificaçoes pulmonares em vidro fosco, bilaterais, periféricas envolvendo lobos inferiores (Shi, et al., 2020).

Apresentamos o caso de um homem de 51 anos de idade, internado em hospital público de referência do Nordeste do Brasil para casos graves de COVID-19, com prognóstico de gravidade e comorbidade autorreferida de obesidade grau II.

\section{Metodologia}

Este estudo é do tipo relato de caso com análise documental.

O relato de caso é uma fonte de informação muito importante que podem subsidiar situações que podem melhorar o tratamento de pacientes em determinadas patologias (Yoshida, 2007). A utilização da análise documental justifica-se pela utilização de materiais que ainda não receberam tratamento analítico e que podem ser reorganizados para compor o objetivo de pesquisas (Gil, 2008). 
As informações contidas neste estudo foram obtidas por meio da revisão do prontuário, relatórios de evolução médica e de Enfermagem, exames laboratoriais e Tomografia Computadorizada.

Os dados coletados e analisados são referentes aos dias 01 a 09 de maio de 2020, durante o período de internamento do paciente com obesidade grau II em um Hospital Público, para tratamento da COVID-19.

O Estudo obedeceu aos preceitos que norteiam a Resolução nº 466/2012, de 12 de dezembro de 2012 do Conselho Nacional de Saúde/Ministério da Saúde (Brasil, 2012); apresentou termo de anuência da instituição hospitalar; a dispensa do Termo de Consentimento Livre e Esclarecido por se tratar de auto-relato; e os critérios da Resolução 1098 de 30/03/1983 Declaração de Helsinque (Associação Médica Mundial 1964), sendo aprovado pelo Comitê de Ética em Pesquisa da Universidade Federal do Cariri - UFCA, sob o Parecer n ${ }^{\circ} .236 .883$.

\section{Apresentação do Caso}

Paciente do sexo masculino, 51 anos de idade, apresentou tosse seca em 21 de abril de 2020; iniciou tratamento com Azitromicina $600 \mathrm{mg}$ de $12 / 12 \mathrm{hs}$ e prednisona $20 \mathrm{mg}$ por cinco dias; no terceiro dia da doença, manteve saturação em $94 \%$ e apresentou febre $\left(38^{\circ} \mathrm{C}\right)$ cedendo com dipirona 500mg; no quinto dia de tratamento a febre retornou, sentiu-se adinâmico e cansado ao realizar pequenos esforços; procurou uma Unidade de Pronto Atendimento, onde recebeu diagnostico médico de COVID-19 com base nos sinais e sintomas; iniciou uso de fosfato de oseltamivir $75 \mathrm{mg}$ e realizado o teste Swab, positivado após 15 dias dos primeiros sintomas.

Do oitavo ao décimo dia da doença, a saturação caiu de $94 \%$ para $88 \%$. No $10^{\circ}$ dia realizou Tomografia Computadorizada que evidenciou comprometimento de mais de $75 \%$ dos pulmões. No dia seguinte $\left(11^{\circ}\right.$ dia da doença), foi admitido em Hospital de Referência para pacientes com quadro grave de COVID-19, onde foi acompanhado por equipe multiprofissional, recebendo alta hospitalar após 9 dias de permanência.

A detecção de COVID-19 foi diagnosticada por meio de sinais e sintomas apresentados e confirmado pelo resultado positivo do gene ORF1ab da SARS-CoV-2 por coleta com Swab nasofaríngeo, colhido no quinto dia da presença de febre e tosse e, posteriormente por Tomografia Computadorizada (Figura 1).

Figura 1 - Imagem da Tomografia Computadorizada em padrão vidro fosco. Fortaleza, Ceará, 2020.
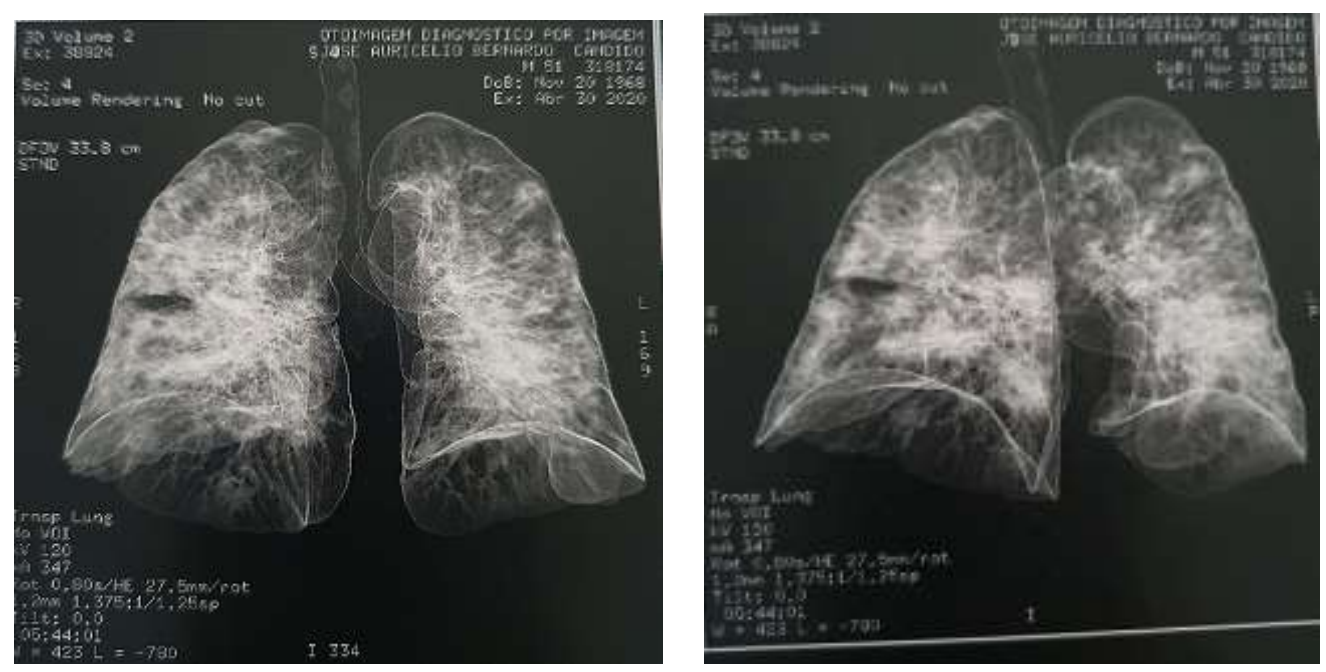

Fonte: Exame de imagem fornecido pelo próprio paciente. 
A Tomografia Computadorizada (TC) foi realizada com tomógrafo MULTISLICE por meio de aquisições volumétricas seguidas por reformatações multiplanares em Workstation, sem contraste.

Pela análise, observaram-se múltiplas opacidades com atenuação predominante de "vidro fosco" distribuídas em campos superiores e inferiores de ambos os pulmões, sobretudo nas periferias póstero-basais, com espessamento dos septos interlobulares em permeio ("pavimentação em mosaico"), áreas de consolidação e faixas de distorção arquitetural pulmonar. Essas alterações comprometem em mais de 75\% da área total dos pulmões em análise visual.

Presença de calcificação em linfonodos no mediastino e hilo esquerdo de aspecto residual. Estruturas vasculares mediastinais sem alterações. Não foram observadas linfonodomegalias mediastinais ou hílares. Hilos pulmonares sem anormalidades perceptíveis. Traqueia e brônquios permeáveis e de diâmetros normais. Vascularização pulmonar normal. Estruturas componentes da parede torácica de aspecto normal.

Em relação às manifestações clínicas o paciente apresentava $\mathrm{IMC}=36,1$ obesidade grau II; percebeu-se exacerbação dos sinais e sintomas nos três primeiros dias de internamento: febre $-38,0^{\circ} \mathrm{C}, 38,2 \mathrm{C}$ e $38,3^{\circ} \mathrm{C}$; PAM $88,5 \mathrm{mmHg}, 85 \mathrm{mmHg}$ e 95 mmHg; PO2 e CO2 mantiveram-se em 36\%; FC - 76 bpm, 92bpm, 86bpm; FR - 24mrm, 19mrm, 16mrm; SatO2 - 95\%, 94\%, 95\% com suporte de oxigênio por cateter em 1litro/min.

Os exames laboratoriais evidenciaram um processo inflamatório mais exarcebados também durante os três primeiros dias de internamento: Hemoglobina (HB) - 15,6g/Dl, 15,7g/Dl e 14,9g/Dl; Hematócrito (HT) - 45,6\%, 45,0\% e 45,0\%; Leucócitos (Leuco) - 4.700 $\mathrm{mm}^{3}, 6.420 \mathrm{~mm}^{3}$ e $6.940 \mathrm{~mm}^{3}$; porém evidenciou-se DHL - 865U/L, 499U/L, 562U/L; PCR $35 \mathrm{mg} / \mathrm{dl}, 95 \mathrm{mg} / \mathrm{dl}, 136 \mathrm{mg} / \mathrm{dl}$; e Linfocitopenia $-564 \mathrm{~mm}^{3}, 661 \mathrm{~mm}^{3}$ e $690 \mathrm{~mm}^{3}$, respectivamente. Após o período de internamento observou-se uma melhora dos exames laboratoriais HB - 15,1g/Dl; HT - 47,6\%; Leuco - 8.629MM ${ }^{3}$; DHL 375U/L; PCR - 1,56mg/dl e Linfócitos - 1.555 $\mathrm{mm}^{3}$.

\section{Discussão}

Durante a pandemia da COVID-19 diversos fatores foram associados à doença decorrente do desconhecimento fisiopatológico da infecção virais o que buscou o aprofundamento seletivo para uma nova doença mundial abordando os achados clínicos de sinais e sintomas, estratificando a gravidade da doença e classificando as pessoas como assintomáticas e sintomáticas, portadoras da doença mais leve, grave e muito grave que exijam hospitalização e casos fatais (Lipsitch, Swerdlow \& Finelli, 2020).

A indicação para a realização de exames baseia-se nos sintomas preliminares como febre nova e alterações no trato respiratório como tosse e dispnéia ou que viajaram para área de transmissão comunitária nos últimos 14 dias ou tiveram contato com pessoas infectadas, bem como pessoas com mais de 60 anos de idade ou que apresentem comorbidades (Hussain, Bhowmik \&, Moreira, 2020).

Percebe-se que os testes para a doença em casos suspeitos são muito limitados devido a pouca quantidade para testar toda a população. O teste mais adequado no momento é o RT-PCR que detecta o ácido nucléico positivo para o SARS-COV-2 no escarro, na garganta e nas secreções do trato respiratório inferior, por meio de SWAB (Organização Mundial da Saúde, 2020).

De acordo com os achados em estudos anteriores, a TC de tórax de paciente com COVID-19 com comprometimento pulmonar mostra opacificaçoes em vidro fosco com ou sem anormalidades consolidadas e está propensa a bilateralidade pulmonar periférica envolvendo lobos inferiores e podem evidenciar os achados antes mesmo do aparecimento dos sintomas (Shi et al., 2020). Apresentam, ainda, características típicas que podem ser uteis na triagem precoce de casos suspeitos enquanto aguardam os exames confirmatórios, pois evidencia a extensão da lesão e os casos mais graves da doença. 
Em estudo recente com 101 casos de pneumonia COVID-19 realizados em quatro instituições de saúde em Hunan, na China, percebeu-se opacidades em vidro fosco em $n=87(87,1 \%)$, misto em $n=53(52,5 \%)$ e consolidação $n=65$ (64,4\%); além disso, percebeu-se comprometimento vascular dos pulmões $n=72$ (74,3\%) e bronquiectasia de tração $n=53$ (52,5\%). As evidencias mostraram distribuições periféricas das lesões, envolvimento bilateral com predominância inferior e multifocal (Zhao, Zhong, Xie, Yu \& Liu, 2020).

Em outro estudo, a TC mostrou uma sensibilidade de $92 \%$, especificidade de $84,4 \%$ e precisão de $79,1 \%$ para detecção da doença e propõe que o exame seja incorporado como teste de triagem para auxiliar a otimização dos testes de PCR, leitos de isolamento e de Unidade de Terapia Intensiva durante a pandemia de COVID-19 (Barbosa, Bittencourt, Miranda, Almeida \& Chojiniak, 2020).

Em relação às manifestações clínicas o paciente apresentava IMC $=36,1$ obesidade grau II; percebeu-se exacerbação dos sinais e sintomas nos três primeiros dias de internamento: febre $-38,0^{\circ} \mathrm{C}, 38,2 \mathrm{C}$ e $38,3^{\circ} \mathrm{C}$; PAM $88,5 \mathrm{mmHg}, 85 \mathrm{mmHg}$ e 95 mmHg; PO2 e CO2 mantiveram-se em 36\%; FC - 76 bpm, 92bpm, 86bpm; FR - 24mrm, 19mrm, 16mrm; SatO2 - 95\%, 94\%, 95\% com suporte de oxigênio por cateter em 1litro/min.

Neste estudo observou-se a persistência da febre, tosse, mialgia e cansaço físico aos pequenos esforços. Esses sintomas foram evidenciados em vários estudos (Wang, Horby, Hayden \& Gao, 2020) porém alguns sintomas como anosmia e disgeusia foram encontrados e pouco relatados (Giacomelli, Pezzati, Conti, Bernacchia, Siano \& Oreni, 2020).

Os mecanismos fisiopatológicos ainda estão sendo acompanhados em todo o mundo, porém alguns casos mais graves da COVID-19 foram observados em idosos e pacientes com comorbidades como doenças cardiovasculares, DM, hipertensão, doença renal crônica e câncer (Divella, De Luca, Abbate, Naglieri \& Daniele, 2020). Estudos evidenciam, também, que pacientes com obesidade com IMC elevado têm algum vínculo epidemiológico com os efeitos graves da doença, provavelmente pelo estado inflamatório de baixo grau a que estão propensos previamente pela liberação de citocinas - TNF $\alpha$, IL-1, IL-6 - secretadas pelo tecido adiposo (Huang et al., 2020). A consequiência desse processo inflamatório é o aumento da hipóxia e isquemia que vai acarretar um estresse oxidativo provocando um aumento da secreção das proteínas inflamatórias e de radicais de oxigênio que vão piorar a fisiologia intracelular (Ellulu, Patimah, Khaza'ai, Rahmat \& Abed, 2020).

As informações de comorbidades, sinais e sintomas relacionados aos exames laboratoriais evidenciaram que os exames Desidrogenase Lática (DHL) por ser um marcador e destruição tecidual proporcional ao grau de lesão pulmonar, a Proteína C Reativa (PCR-hs) por ser um marcador inflamatório que define um mau prognóstico de pessoas com SARA e a contagem de linfócitos (Linfocitopenia) por representar a disfunção imunológica associada à forma mais grave das doenças. Esses exames apresentaram uma acurácia de $90 \%$ em estudo que indicou que a análise desses marcadores pode ser utilizada para acompanhar a evolução da COVID-19 a partir dos sintomas. Os valores de referencia DHL > 365 U/L, PCR-hs > 41,2 $\mathrm{mg} / \mathrm{L}$ e linfócitos totais $<14,7 \%$, indicam maior risco de gravidade e mortalidade (Rodríguez-Morales, MacGregor, Kanagarajah, Patel, \& Schlagenhauf, 2020).

\section{Conclusão}

Nesse relato de caso, a infecção por COVID-19 se comportou de forma persistente. A obesidade como única comorbidade detectada, pareceu ser contribuinte para a demora na recuperação e alta. Percebeu-se que tanto na obesidade como na COVID-19 os processos inflamatórios são comuns e evidenciados pelos mesmos marcadores; esses processos podem exacerbar a infecção causada pelo SARS-CoV-2. Devido ao crescente volume de pesquisas relacionadas à pandemia, estudos futuros deverão apontar evidências concretas quanto à relação entre a obesidade e a COVID-19.

Espera-se que este estudo possa contribuir com estudos científicos e acadêmicos nos delineamentos da ciência investigativa sobre outros determinantes sociais e de vulnerabilidade para a COVID-19. 


\section{Referências}

Associação Médica Mundial (1964). Resolução 1098 de 30 de junho de 1983 -Declaração de Helsinque - Adotada pela $18^{a}$ Assembléia Mundial de Médicos, Helsinque, Finlândia, 1964. https://www.fcm.unicamp.br/fcm/sites/default/files/declaracao_de_helsinque.pdf.

Barbosa, P. N. V. P., Bittencourt, A. G. V., Miranda, G. D., Almeida, M. F. A. \& Chojiniak, R. (2020). Chest CT accuracy in the diagnosis of SARS-CoV-2 infection: initial experience in a cancer center. Radiol Bras, http://www.scielo.br/scielo.php?script=sci_arttext\&pid=S0100-39842020005004210\& $\operatorname{lng}=$ en $\&$ nrm=iso.

Brasil (2012). Ministério d Saúde, Conselho Nacional de Saúde. Resolução $n^{o}$ 466, de 12 de dezembro de 2012. Dispõe sobre diretrizes e normas regulamentadoras de pesquisas envolvendo seres humanos. Diário Oficial [da] República Federativa do Brasil, Brasília, DF. http://bvsms.saude.gov.br/bvs/saudelegis/cns/2013/res0466_12_12_2012.html.

Brasil (2020). Ministério da Saúde. Protocolo de manejo clínico do coronavírus (COVID-19) na atenção primária à saúde: versão 9 (maio de 2020). Secretaria de Atenção Primária à Saúde. Brasília - DF. 2020. http://portalarquivos2.saude.gov.br/images/pdf/2020/May/05/20200504-ProtocoloManejover09.pdf.

Dietz, W. Santos-Burgoa, C. Obesity and its Implications for COVID-19 Mortality. Obesity (Silver Spring). 28(6):1005. 10.1002/oby.22818. https://pubmed.ncbi.nlm.nih.gov/32237206/.

Divella, R., De Luca, R., Abbate, I., Naglieri, E. \& Daniele, A. Obesity and cancer: the role of adipose tissue and adipo-cytokines-induced chronic inflammation. J Cancer. 2016;7(15):2346-2359. 10.7150/jca.16884. https://pubmed.ncbi.nlm.nih.gov/27994674/.

Ellulu, M. S., Patimah, I., Khaza'ai, H., Rahmat, A. \& Abed, Y. (2017). Obesity and inflammation: the linking mechanism and the complications. Arch Med Sci. 2017;13(4):851-863. 10.5114/aoms.2016.58928. https://www.ncbi.nlm.nih.gov/pmc/articles/PMC5507106/.

Giacomelli, A., Pezzati, L., Conti, F., Bernacchia, D., Siano, M. \& Oreni L. Self-reported olfactory and taste disorders in SARS-CoV-2 patients: a crosssectional study. Clin Infect Dis. 2020 10.1093/cid/ciaa330. https://pubmed.ncbi.nlm.nih.gov/32215618/.

GIL, A. C. Métodos e técnicas de pesquisa social. (6a ed.) Atlas, 2008.

Guan, W. J., Ni, Z. Y., Hu, Y., Liang, W. H., Ou, C. Q. \& He, J. X. (2020). Características clínicas da doença por coronavírus 2019 na China. N Engl J Med. 2020: 1-13. https://pubmed.ncbi.nlm.nih.gov/32109013/.

Huang, C., Wang, Y., Li, X., Ren, L., Zhao, J., Hu, Y., Zhang, L., Fan, G., Xu, W., Xie, X., Yin, W., Li, H., Liu, M., Xiao, Y., Gao, H., Guo, L., Xie, j., Wang, G., Jiang, R., Gao, Z., Jin, Q., Wang, J. \& Cao, B. (2020). Clinical features of patients infected with 2019 novel coronavirus in Wuhan, China. Lancet. 2020;395(10223):497-506. 10.1016/S0140-6736(20)30183-5. https://pubmed.ncbi.nlm.nih.gov/31986264/.

Hussain, A, Bhowmik, B. \&, Moreira, N. C. V. (2020). COVID-19 and diabetes: Knowledge in progress. Diabetes Res Clin Pract. 2020;162:108142. 10.1016/j.diabres.2020.108142. https://pubmed.ncbi.nlm.nih.gov/32278764/.

Kassir, R. Risk of COVID-19 for patients with obesity. Obes Rev. 2020;21(6):e13034. 10.1111/obr.13034. https://pubmed.ncbi.nlm.nih.gov/32281287/.

Lipsitch, M., Swerdlow, D. L. \& Finelli, L. (2020). Defining the Epidemiology of Covid-19 - Studies Needed. N Engl J Med. 2020;382(13):1194-1196. 10.1056/NEJMp2002125. https://pubmed.ncbi.nlm.nih.gov/32074416/.

McIntosh, K. (2020). Novel Coronavirus (2019-nCov). UpToDate Jan 2020. https://www.uptodate.com/contents/coronavirus-disease-2019-covid-19epidemiology-virology -e-prevenção.

Organização Mundial da Saúde (2020). Orientação técnica da doença de coronavírus (COVID-19): Testes laboratoriais para 2019 -nCoV em humanos 2020. [30/03/2020]. https://www.who.int/emergencies/diseases/novel-coronavirus-2019/technical-guidance-publications.

Rodríguez-Morales, A. J., MacGregor, K., Kanagarajah, S., Patel, D. \& Schlagenhauf, P. (2020). Going global - Travel and the 2019 novel coronavirus. Travel Med Infect Dis. 2020;33:101578. 10.1016/j.tmaid.2020.101578. https://pubmed.ncbi.nlm.nih.gov/32044389/.

Sattar, N., McInnes, I. B. \& McMurray, J. J. V. Obesity a Risk Factor for Severe COVID-19 Infection: Multiple Potential Mechanisms Circulation. 2020; 10.1161/CIRCULATIONAHA.120.047659.10.1161/CIRCULATIONAHA.120.047659. TIONAHA. 120.047659 . https://www.ahajournals.org/doi/pdf/10.1161/CIRCULA

Shi, H., Han, X., Jiang, N., Cao, Y., Alwalid, O., Gu, J., Fan, Y. \& Zheg, C. Radiological findings from 81 patients with COVID-19 pneumonia in Wuhan, China: a descriptive study. Lancet Infect Dis. 2020;20(4):425-434. 10.1016/S1473-3099(20)30086-4. https://pubmed.ncbi.nlm.nih.gov/32105637/.

Wang, C., Horby, P. W., Hayden, F. G. \& Gao, G. F. (2020). A novel coronavirus outbreak of global health concern. Lancet. 2020;395(10223):470-473. 10.1016/S0140-6736(20)30185-9. https://pubmed.ncbi.nlm.nih.gov/31986257/.

Wu, Z. \& McGoogan, J. M. Características e lições importantes do surto de doença por coronavírus 2019 (COVID-19) na China: resumo de um relatório de 72314 casos do centro chinês de controle e prevenção de doenças. JAMA, J Am Med $323 \quad 10.1001 /$ jama.2020.2648. https://jamanetwork.com/journals/jama/fullarticle/2762130.

Yoshida, W. B. A redação científica. $J$ Vasc Bras. 2007;6(2):112-113. https://www.scielo.br/j/jvb/a/vnKt5ttNpdFMjf6dLcmnM4Q/?format=pdf\&lang=pt.

Zavascki, A. P. \& Falci, D. R. Clinical Characteristics of Covid-19 in China. N Engl J Med. 2020;382(19):1859. 10.1056/NEJMc2005203. https://pubmed.ncbi.nlm.nih.gov/32220202/.

Zhao, W., Zhong, Z., Xie, X., Yu, Q. \& Liu (2020) J. Relation Between Chest CT Findings and Clinical Conditions of Coronavirus Disease (COVID-19) Pneumonia: A Multicenter Study. American Journal of Roentgenology 2020 214: 5, 1072-1077. https://www.ajronline.org/doi/10.2214/AJR.20.22976. 